\title{
Macbeth in der Forensik
}

\author{
Franz Triebenecker ${ }^{1} \cdot$ Stefan Orlob ${ }^{2,3}$
}

Eingegangen: 21. August 2020 / Angenommen: 27. August 2020 / Online publiziert: 30. September 2020

(c) Der/die Autor(en) 2020

\section{Zusammenfassung}

Der psychiatrische Raum kennt viele unterschiedliche Zimmer und Korridore. Es gibt erhebliche Unterschiede zwischen den verschiedensten Kliniken und Institutionen. Eine offene Tagesklinik ist nicht vergleichbar mit dem geschlossenen Maßregelvollzug. Beide nutzen aber bisweilen Methoden, die das Theater bereitstellt. Dabei reicht die Spannbreite von einfachen, improvisierten Rollenspielen bis hin zu professionellen, technisch aufwendigen Inszenierungen vor einem kritisch interessierten Publikum. Zentrale Fragen sind dabei, wie wirkt Theaterspielen, kann es tatsächlich heilen, und wie verhält sich der künstlerische Anspruch zur Therapie? Diese will der nachfolgende Beitrag anhand mehrjähriger praktischer Erfahrungen in einer Maßregelvollzugsklinik aufgreifen.

Schlüsselwörter Theatertherapie $\cdot$ Resozialisierung $\cdot$ Behandlung $\cdot$ Maßregelvollzug

\section{Macbeth in forensics}

\begin{abstract}
The psychiatric field has many different rooms and corridors. There are substantial differences between the very different clinics and institutions. An open day clinic is not comparable with a closed forensic commitment institution; however, both occasionally use methods that appropriate drama therapy. This spectrum ranges from simple improvised role playing to professional technically complex theatrical productions in front of a critically interested public. In this context the core questions are how does drama therapy work, can it actually cure and what effects do the artistic requirements have on treatment? These questions are dealt with in this article based on many years of experience in a forensic commitment clinic.
\end{abstract}

Keywords Drama therapy $\cdot$ Resocialization $\cdot$ Treatment $\cdot$ Forensic commitment

Dem Drama ist die Verkettung von Schuld und Unschuld eingeschrieben. Es lebt „,von der Unmöglichkeit, in Unschuld zu leben“. Die Figuren nehmen in unterschiedlichsten Konstellationen Schuld auf sich, und dies wird im Drama exemplarisch verhandelt. Damit zielt es auf kathartische Wirkungen beim Zuschauer. Die Psyche des darstellenden Schauspielers interessiert da kaum.

\footnotetext{
Dr. Stefan Orlob

stefan.orlob@uni-greifswald.de

1 Kreisdiakonisches Werk Stralsund, Kulturkirche St. Jacobi, Jacobiturmstr. 28a, 18439 Stralsund, Deutschland

2 Psychiatrische Sachverständigenpraxis, Langenstraße 24a, 18439 Stralsund, Deutschland

3 Universität Greifswald, Greifswald, Deutschland
}

Ganz anders ist es, wenn innerhalb eines forensischen Settings Theater gespielt wird. Wird in einer forensischen Klinik eine Bühne aufgeschlagen, auf der Patienten spielen, so rücken der Schauspieler und seine therapeutischen Perspektiven in den Fokus. Die Katharsis des Zuschauers tritt hinter die Wirkungen im Spieler zurück. Die Bühne wird zu einem Ort der Arbeit an sich selbst.

Im Maßregelvollzug ist dies immer noch etwas Besonderes, auch wenn andere Formen der Kunsttherapie (Orlob et al. 1998) eine lange Tradition haben. Ironischerweise findet es hier an seine Ursprünge zurück. Der Erste, der öffentlich in der Psychiatrie inszenierte, war der Marquis de Sade. In der Forensischen Psychiatrie des Helios Hanseklinikums Stralsund ist in Kooperation mit dem Kreisdiakonischen Werk Stralsund genau dies geschehen. In einer Kontinuität 
von nunmehr bald 6 Jahren wurde Theater innerhalb der forensischen Klinik erarbeitet und in öffentlichen Räumen gezeigt. Die Themen waren u. a. die 7 Todsünden, Macbeth, das Gebot „Du sollst nicht begehren“ und Dostojewskis Schuld und Sühne (Triebenecker 2018).

Was geschieht, wenn solche Themen von Patienten der Forensik oder Gefangenen im Strafvollzug (Wißner 2018) öffentlich präsentiert werden? Was geschieht, wenn Täter Täter spielen und dies öffentlich zeigen? Man kann fast bedingungslos voraussetzen, dass die Motivation zur Tat bei den Spielern eher nicht so pathetischer Natur war, wie in den zu spielenden Rollen. Im realen Leben wird ihre Schuld viel banalere, egoistischere, nichtigere und in ihrer psychischen Erkrankung wurzelnde Gründe haben. Das macht die Tat nicht kleiner, sondern eher größer, schuldhafter. Gleichzeitig sind sie Patienten und haben Anspruch und eine Hoffnung auf Heilung. Was also geschieht mit ihnen, wenn sie öffentlich in professionell organisierten und präsentierten Theateraufführungen den Macbeth, den Ödipus oder gar den Marquis de Sade, den Inbegriff des Bösen, geben?

Die Bühne soll ein Ort der Heilung werden. Kann sie das? Die Antwort ist: Die Bühne ist ein Ort der Heilung. Gerade wenn sie öffentlich ist, und sie ist nur als öffentliche Bühne Bühne. Bis ins Paradox spitz formuliert: Theatertherapie muss Kunst sein wollen, um therapeutisch sein zu können, obwohl sie weiß, dass sie keine Kunst sein kann. Diese Paradoxien gilt es zu erklären bzw. aufzulösen, um die therapeutischen Dimensionen der Theaterarbeit aufzeigen zu können. Dabei bewegt sich die Argumentation oft im Kreis, wie eine Schlange, die sich in den Schwanz beißt. Aber auch sie wird letztlich satt und kann den Verlust, zumindest argumentativ, verwinden.

Theater in der Forensik steht unter der Zielstellung, einen Heilungsprozess zu unterstützen. Bevor es gefällt, muss es nutzen. Und zwar keinem Spielleiter, Therapeuten oder $\mathrm{Zu}$ schauer, sondern dem Patienten. Aus der Therapie mit theatralischen Mitteln wird Theater mit therapeutischen Absichten, wenn der Prozess auf eine Aufführung vor Publikum zielt, und wenn der Patient nicht mehr sich selbst und Alternativen zu sich selbst spielt, sondern anderes. Wie bei jedem Schauspieler spielt der theaterspielende Patient nicht mehr sich selbst. Er spielt mit sich als Material, mit geeigneter Mimik, Gestik, Sprechweise und Körpersprache, ihm Fremdes. Deutet so die darzustellende Figur. Zwischen ihn und seine Darstellung ist eine ästhetische Distanz getreten. Mit zwei Schritten wird aus dem Therapeuten ein Regisseur und aus dem Patienten ein Schauspieler. Mit dem ersten Schritt verlässt der Patient das reine Sich-Selbst und stellt mit sich als Material Fremdes dar. Mit dem zweiten Schritt verlässt er die geschlossenen Räume der Therapie und tritt auf die offene Bühne.
Alles, auch das theatrale Gelingen und Wirken, muss dem Patienten dienen. Die Wahl jedes Mittels hat sich an ihm zu bewähren. Er bestimmt das Tempo, den Rhythmus, die innere und äußere Gestalt der Arbeit. Er tut dies aber nicht durch sein ,so sein, wie er ist“", sondern durch das, was er in der Arbeit werden könnte. Der Patient ist ein konstruierender Pol der Arbeit. Er ist zugleich Ausgang und Ziel. Die Aufgabe des Regisseurs ist es, mit ihm das Spiel zu entwickeln. Dies ist kein von Harmonie geprägter Prozess. Das Spiel ringt mit seinen Spielern um Qualität und lässt in der Kreuzung künstlerischer Mittel und therapeutischer Notwendigkeiten ganz Eigenes entstehen.

Bislang richtete sich die Wirkung therapeutischer Arbeit nach innen, auf den Patienten. Jetzt richtet sie sich nach auBen auf das Publikum. Gleichzeitig bleibt der Patient Ziel der Arbeit. In der Produktion soll er erfahren, was Rollenspiel, Perspektivwechsel, Kreativitäts- und Souveränitätsgewinn und anderes ermöglichen. Dies bleibt aber nicht als Übung stehen, sondern passt sich in ein kollektives Ringen um Darstellung ein. Damit wendet er sich dem Publikum zu. Jetzt werden die Binnenperspektiven aufgebrochen und in der Präsentation nach außen gewendet. Die Therapie verwandelt sich in Theater, die Patienten in Darsteller und der Therapeut in einen Regisseur.

Dabei ist von der ersten Begegnung der Fokus auf die öffentliche Präsentation gerichtet. Durch dieses Bekenntnis zur Öffentlichkeit ist selbst im anfangs geschützten Raum der Probe permanent ein fremder Blick vorhanden. Und so, wie die Anwesenheit der Zuschauer bei der Vorstellung das Binnengefüge des Ensembles verändert, so verändert die Perspektive des fremden Blicks in der Probe das Verhaltensgefüge des Spielers. Schon jetzt ist der fremde $\mathrm{Zu}$ schauer Katalysator und Verstärker. Seine Anwesenheit und Intensität werden im fortschreitenden Probenprozess immer stärker, bis er in der gefühlten Omnipräsenz im letzten Dunkel vor dem sich hebenden Vorhang gipfelt.

In diesem Moment schießt all das zusammen, was bis hierher erarbeitet und vorausgedacht wurde. Alle Pannen und kleinen Erfolge, alle Erfahrungen und Zuversicht wachsen zu etwas ganz Neuem zusammen. In der Begegnung mit dem Zuschauer entsteht etwas, das Patienten und Zuschauer bereichert und den Patienten u.U. langfristig neue verändernde Erfahrungen machen lässt. Je fantasievoller, ästhetisch überwältigender diese Begegnung für beide wird, umso nachhaltiger das Erlebnis und Ergebnis für den Patienten. Der Schritt aus dem Therapiezimmer auf die beleuchtete Bühne ist vielleicht therapeutisch motiviert, muss aber auch künstlerisch überzeugend sein. Das Private wendet sich jetzt ins Öffentliche. Aus dem Sichzeigen in der Therapie wird das Sichzeigen vor anderen in der Fiktion der theatralen Gesamtkonstruktion einer Aufführung.

Dies ist nicht ungefährlich, denn für die auf der Bühne Stehenden bergen diese Momente existenzielle Risiken. 
Erfüllen sich ihre Ängste und bestätigen sich ihre Zweifel, wird das Darstellen auf der Bühne nicht nur Scheitern, sondern in peinliche Selbstenthüllung umschlagen. Sie geben sich dem Publikum anheim und sollten von dessen Reaktionen geschützt werden. Dieser Schutz liegt im künstlerischen Gelingen. Dabei gilt nicht das reine Gefallen oder Missfallen des Publikums, sondern die intensive theatralische Auseinandersetzung. Deswegen gilt unterhalb des Einspruchsrechtes des Therapeutischen die Autonomie des Ästhetischen. In ihr müssen die theatralische Konstruktion und die Spielführung des Regisseurs so stark sein, dass sie dem Spielenden nicht nur Schutz gewährt, sondern ihn erhebt und auf ein Podest stellt. Der Spielende selbst schafft jetzt den glückhaften, berauschenden Moment des öffentlichen Gelingens.

Um dies zu ermöglichen, muss der Regisseur eine eigene Ausdrucks- und Spielweise entwickeln, die nicht mit dem institutionalisierten Illusionstheater wetteifert. Vielmehr gilt es, die Persönlichkeit der Spielenden und ihr ureigenstes Ausdruckspotenzial zu entdecken und dies zu einer eigenen theatralischen Ausdrucksweise in der künstlerischen Perspektive des Regisseurs entwickeln. Die Faszination für den Zuschauer entsteht durch die spezifische subjektive ins Bild gesetzte Expressivität des Spielers.

Die entscheidende Differenz zum professionellen Theater bleibt ein finales Einspruchsrecht des Therapeutischen gegen das Ästhetische. Es kann schon sein, dass hier und da nicht gehen darf, was in der ästhetischen Perspektive notwendig gewesen wäre, um das Ganze gut werden zu lassen. Die mögliche Beschädigung eines Spielers verbietet dies. Das heißt für den Regisseur, nicht zu verzichten, sondern weiterzusuchen, andere Ideen zu probieren. Gerade hier zieht sich die Fantasie des Regisseurs nicht vor der Therapie zurück. Im Dialog mit ihr wird seine, für das psychiatrische Setting andere, Professionalität deutlich und herausgefordert. Solche mit sich ringenden Prozesse verlaufen weder kontinuierlich noch harmonisch. Sie sind vom ständigen Scheitern bedroht, jedoch enorm produktiv. Bei allen Einschränkungen der Patienten als Spieler oder aller Exklusivität des Spielortes muss der therapeutische Ansatz in der Wahrnehmung des Zuschauers zum Verschwinden gebracht werden. Wichtig ist jetzt nicht mehr, wer spielt, warum oder wo gespielt wird, sondern allein, dass und wie gespielt wird. Der Zuschauer hat das Recht auf die Priorität des Ästhetischen in der eigenen Wahrnehmung. Der spielende Patient hat ein Recht auf die permanente Berücksichtigung des therapeutischen Einspruchs.

Die Veröffentlichung theatertherapeutischer Arbeiten als Inszenierung für Publikum verändert nicht nur das innere Beziehungsgeflecht zwischen Spielern und Anleitern. Auch die Zuschauer schauen anders auf diese "Schauspieler". Theaterspielende forensische Patienten werden von anderen Gefühlen und Interessen der Zuschauer begleitet als bezahl- te Profis. Hier schwingt immer noch ein wenig Skandal mit. Allein die Tatsache des öffentlichen Agierens wird aus moralisch festen Positionen heraus kritisch gesehen. Patienten und Zuschauer befinden sich dadurch in deutlich exponierteren Positionen als in vielen anderen Situationen. Der Zuschauer befindet sich immer in Nachbarschaft zum Voyeur. Dies bringt Patienten in die Nähe des Objekts, das aus schadenfroher oder beschämender Neugier mit Häme betrachtet wird. Gerade in der Arbeit mit forensischen Patienten ist Entstigmatisierung deshalb immer noch nötig, jedoch auch besonders problematisch. Denn hier sind Patienten immer auch Täter, deren Opfer im Blick sein müssen, will man nicht im Nachhinein die Tat aus ihrem Unrechtszusammenhang lösen.

Andere lehnen solch eine Weise des öffentlichen Produzierens und Präsentierens aus moralischen Gründen ganz ab. Es ist offensichtlich und berechtigt, dass Opfer von Gewalttaten eine andere Perspektive auf solches Treiben haben als Therapeuten und Sozialarbeiter. Lehnen die Ersteren aus einer strafenden Perspektive heraus dies eher grundsätzlich $\mathrm{ab}$, begegnen die anderen solchen Arbeiten mit Sympathie. Man kann sich hier auch nicht aus der Verantwortung ziehen, indem man sagt, dass Theater provoziert und gleichzeitig zur Therapie gehört. Die Emotionen gehen tief und reichen hinab bis in grundsätzliche Auffassungen von Strafe und Sühne im menschlichen Sein. Jede Inszenierung muss den Rahmen dafür selber finden. Ist die Inszenierung ehrlich zu sich selbst und den Zuschauern, so stellt sie sich in der Darstellung zu sich selbst kritisch. Sie hinterfragt szenisch ihre eigenen Fundamente, kennt keine klaren Antworten und gibt auch dem Spieler nicht in allem recht. So können Positionen entstehen, die trotz einer therapeutischen Grundrichtung zwischen Spielern und Zuschauern keinen sozial-moralischen Frieden herstellen, welchem sich das ästhetische Handwerk unterordnet. Das kritische Hinterfragen der eigenen Position ermöglicht es, dass auch mit diesen Darstellern das Hier und Jetzt verhandelt werden kann, und somit auch die Dimensionen ihres eigenen Handelns in dieser Gegenwart.

Seine Berechtigung hat Theater im Komplex von Tat und Strafe nur, wenn die dem geltenden Recht zugrunde liegende Menschensicht auf Integration nach Strafe setzt und nicht Strafe als Rache verlangt. Mit dem Ziel einer späteren Reintegration und der Verantwortungsübernahme des Täters für seine Tat, sich selbst und seinen Mitmenschen gegenüber, werden Therapie und damit Theater in der Therapie möglich. Vergäße die theatrale Arbeit diese Basis, würde sie zu einem Instrument der Verdrängung und nicht der Selbstreflexion. Deswegen sind gerade Stoffe, die sich den Themen um Schuld, Strafe und Sühne zuwenden, prädestiniert für diese Arbeit. Macbeth ist eben die Geschichte einer Tat, die die Opfer und die Täter zugrunde richtet. Immer wieder stöhnt unser Macbeth: „Ich tat die Tat, was tut sie mir.“ 
Theater ist immer Arbeit mit sich selbst am Fremden. Der Spieler spielt mit sich selbst, mit seinen Erfahrungen, seiner Leiblichkeit, seinen Gefühlen den ihm fremden Macbeth, einen schottischen mittelalterlichen adligen Krieger, der bis zu einem bestimmten Punkt stolz und herrschaftlich auf seine Stärke zur Gewalt schaut. Er entdeckt diesen anderen, indem er ihn aus sich selbst und mit sich selbst konstruiert. Damit begreift er Mechanismen, erkennt Strukturen und Motive des Begehrens, Verstrickungen. Die Brücke zwischen sich selbst und dem anderen ermöglicht ihm der Regisseur, aber er allein geht und entwirft die andere Seite. Diese Konstruktionsarbeit wirft natürlich auch den Blick zurück auf das eigene Werden und Sein und führt auch hier zu neuen Konstellationen und Sichten. Und dies in einem Prozess, der von Empathie für die Rolle und den Mitspieler, von Verantwortung und Selbstverantwortung, von Disziplin und Versuch geprägt ist.

Für den Zuschauer darf dies nicht in Wahrnehmung der therapeutischen Absicht und pädagogischer Zwänge enden, sondern in einem spannenden Theaterabend. Ob es gelingt, die therapeutische Absicht in die Sinnlichkeit des inspirierten Zuschauens zu transformieren, liegt in der Fähigkeit des Spielers und der Inszenierung, Imaginationen zu provozieren. Die Inszenierung muss das Gegebene durch theatrale Konstruktion überschreiten. Nicht die Präsentation des Spielers ist das Ziel, sondern die Imagination durch den Spieler. Ziel ist nicht Dokumentation des Seienden, sondern dessen fiktive Infragestellung, nicht wirkliche Authentizität, sondern Arbeit mit dem Schauspieler, der sich selbst als Material behandelt. Dabei wird nicht das eigene Leben verbrämt oder exemplarisch thematisiert, sondern mit dem Eigenen auch das Ganze infrage gestellt. Macbeth ist nicht nur die Figur Macbeth und der ihn darstellende Spieler; er ist auch die Geschichte gesellschaftlicher Muster und Widersprüche, die von allen Spielern und ihrer Inszenierung zusammen mit sich selbst infrage gestellt werden.

Wenn Theater sich in psychiatrische oder andere lebensweltliche fragile Räume begibt, soll es bei allen notwendigen Kompromissen an die Lebenswelt nichtbezahlter Amateure Theater bleiben. Nur so kann es bei Spielern und Zuschauern das freisetzen, was Theater so fruchtbar für Therapie macht. Sich selbst infrage zu stellen, in der Distanz Wirklichkeit zu begreifen, um zumindest die Chance auf Veränderung zu haben, ist ein gemeinsamer Grundzug von Theater und Therapie. In gegenseitiger Verklammerung und Rücksichtnahme gilt es, dies zu produzieren. Weder Patienten noch Therapeuten noch Künstler sind dabei in ihren jeweiligen Seins- und Arbeitsweisen zu funktionalisieren oder sollten auf das Ihrige verzichten. Nur das konsequente Ringen um die eigene Ausdrucksweise bringt die Positionen zusammen. Was wie ein schlechter Kompromiss erscheint, ist die dem Theater eigene Produktionsweise des miteinander Ringens der unterschiedlichen Kompetenzen und subjektiven Perspektiven. Diese Prozesse werden von vielen Widersprüchen vorangetrieben und bis zur Premiere krisenhaft erfahren.

Die Bühne eröffnet eine Zeit und einen Raum, in dem das Ich etwas anderes als sich selbst entwerfen kann. Das kann Flucht, Traum oder Perspektivwechsel sein. Das andere kann Last oder Lust sein. In jedem Fall gerät das festgefahrene Ich in Bewegung und begibt sich auf einen unbekannten Weg, der im besten Fall therapeutisch ist. Der Einzelne kann sich im theatralen Spiel entgrenzen, anders und neu sein. Er erlebt Weisen des Seins, die ihm nicht möglich erschienen. Er bricht auch im Gefängnis die Mauern seines Ich auf und erlebt auf dem langen Weg über Fantasie und repetierende Disziplin sich selbst als anderen im Rausch der Premiere.

Dies alles lässt sich kaum erzwingen. Erfahrungsgemäß gibt es große Widerstände bei Patienten, sich dem Theaterspiel zu öffnen oder sich gar in der Öffentlichkeit zu zeigen. Allzu oft können sich Patienten gar nicht dem spielerischen Entdecken des Eigenen und des Fremden öffnen. Sie verharren in ihren Positionen und Zwängen. Sie können keine subjektive Freiheit erleben, um sich dem zweckfreien Spiel hinzugeben. Sie haben nicht die persönliche Stärke, um sich in irgendeiner Weise des Spielens frei und spontan bewegen zu können. Bis in die Proben hinein wirken die dominanzsuchenden Rollenmuster der hierarchisch strukturierten sozialen Wirklichkeit der Patienten. Wo objektive Freiheit nicht ist, kann subjektives Freisein kaum möglich werden. Die im Spiel mögliche Freiheit kann ebenso nicht angenommen werden, da fremde Rollenzuweisungen und eigene Zwänge jede nicht zweckhafte Handlung beschränken. Daraus realisiert sich ja der therapeutische Bedarf.

So liegt am Beginn des Theaterspielen in psychiatrischen Settings oft nicht das freie Spiel, sondern das gesprochene Wort, sei es im Gespräch oder als vorliegender Text. Die Aufgaben des Spiels als Beginn sind das Entwickeln von Alternativen im Handeln, die Entwicklung von Lust, das Erlernen intensiver Wahrnehmung und v. a. das Spüren und Zeigen eigener und fremder Gefühle. Dies ist in den meisten Fällen nur schwer möglich, weil Scham und Zweifel jeden Spaß am Entdecken ersticken. Vor allem die eigene Emotionalität wird versteckt, vor den anderen und vor sich selbst. Gefühle zu offenbaren, gilt als Schwäche, sich zu zeigen, als dumm. Damit stehen die Patienten zwischen sich und dem Spiel.

Spielerische Freiheit kann nur das künstlerische und auch therapeutische Ziel des Probenprozesses sein, nicht seine Voraussetzung. Dies ermöglicht ein Spiel, das aus dem Gespräch erwächst und am Anfang von Vorgaben durch den Regisseur geprägt ist. An diesen Vorgaben hangelt sich der spielende Patient bis in das eigene Spiel, in dem er dann auch frei und souverän sein kann. Diese Souveränität ermöglicht das Ablegen der Rollen und 
das Sicherleben und Sichzeigen. Dies wird noch dadurch erschwert, dass wir das Spielmaterial und den Text mit Patienten und aus ihrer Persönlichkeit entwickeln. Vor dem Beginnen gibt es die Idee einer ästhetischen Konzeption. Diese ist mehr Rahmen als Bild. Oft ist es ein Bild der Bühne, die das Spiel bestimmen wird. Manchmal ist es anderes. Bevor sich im Rahmen das endgültige Bild konstruiert, müssen alle Gegebenheiten kennengelernt werden. Wer spielt überhaupt mit? Über welche Ressourcen verfügen die einzelnen Spieler? In der Forensik spielen sicherheitstechnische Aspekte bis in die Dramaturgie und Spielführung eine Rolle. Das ist zum Beginn auch für die spielenden Patienten oft schwierig.

Theater in der Psychiatrie steht in der Spannung zwischen einem klaren therapeutischen Auftrag und einer sinnlichen Verselbstständigung der Aufführungspraxis. Die Beziehung beider Pole aufeinander bleibt dem Theater in der Therapie als widerständiger Prozess eingeschrieben. Wenn ein Pol den anderen negiert, veröden Therapie und Theater in blutleerer uninspirierter Anspannung. Ästhetische Sinnlichkeit und therapeutische Funktion müssen sich widerständig miteinander verschränken. In seinem Rahmen und auf seinen Brettern wird aus der Angst Mut, aus der Einsamkeit im grellen Licht die Gemeinschaft im Applaus, aus der Disziplin der Rausch. Es ist gleichzeitig immer ein Übergang aus der Gruppe in das Einzelne, denn so viel auch in der Gruppe probiert wurde, im Rampenlicht ist jeder zuerst allein. Erst im Schlussapplaus existiert in vertiefter und intensiverer Weise das Erleben der Gemeinschaft. Und dieser Moment ist die Rückverwandlung des Arbeitens mit sich selbst in Sich-Selbst, das jetzt um eine Prägung reicher geworden ist.

Für Patienten ist dies eine neue und auch befremdliche Erfahrung, die sie auch erst machen, wenn sie in das Licht treten. Vorher werden sie von ganz anderen Gedanken beherrscht: Der Schlussapplaus befreit und erlöst von dieser Erfahrung. Vor allem beendet er die Verunsicherung, die da war und welche die ganze Vorstellung begleitet. Diese Erfahrung ist Teil dessen, was beim Spielen als Krise erfahren wird. Sie ist größte Nähe zu sich selbst und gleichzeitig Distanz. In der Fremde wird das Ureigenste intensiv erfahren. Und dass dabei zugeschaut wird, ist selbstverständlich mit Scham besetzt. Man fühlt sich bei einer intensiven intimen Erfahrung beobachtet. Man fühlt sich nackt. Unsere Spieler erfahren dies fast schockhaft, von vielen Ängsten begleitet. Deswegen wird dieser Moment so wichtig und ein zentrales Agens der therapeutischen Effekte. Durch die Dialektik von Figur und Person gibt der Spielende sich selbst eine Bedeutung, die eine andere ist als er selbst, die aber durch seine Kraft erwächst. Er schreibt sich Neues zu und erhebt sich aus der Profanität seines Seins. Er betritt mit der Bühne einen Boden, der ihn, obwohl er er selbst bleibt, größer und anders erscheinen lässt. Die Bühne ist der er- hebende Raum, der Katalysator, mehr nicht. Alles andere leistet der Spieler. Das anwesende Publikum ist Teil und Agens dieser Transformation und vollendet sie durch seinen Applaus. Dazu bedarf es natürlich vorher Anlässen, die im Ringen der ästhetischen Vision des Regisseurs mit den Möglichkeiten, den Fantasien und den Kräften der Darsteller entstehen. Und diese müssen sich selbst an den mehr oder weniger großen Ressourcen der Spieler entzünden.

Vieles, was der Schauspieler als Profi von sich aus macht, muss der Regisseur übernehmen, um das Erspüren des anderen für den Spielenden zu ermöglichen. Er zeigt ihm das Beobachten, das Agieren im Modus des anderen. Der geübte Schauspieler ist immer auch ein geübter Beobachter, der anderes intensiv betrachtet, um es in seiner Darstellung nachahmen oder auch neu herzustellen zu können.

Nun ist das andere, therapeutisch gesehen, oft nicht nur das Fremde, sondern auch das sehr weit Entfernte. Hier muss der Spielleiter zur Brücke für die ersten Schritte auf das andere hinwerden. Diese Brücke schlägt den Weg vom reinen Sich-Selbst zur gespielten Figur hin. Mit dem Verstehen des anderen in der zu spielenden Figur kann das Verstehen des anderen an sich gelernt werden. Der Schritt aus sich selbst hinaus zur Figur gestaltet nicht nur die Figur, sondern auch das eigene Selbst. Als Konstruktion sollte dieser Weg gar nicht aus der Darstellung verschwinden. Die Differenz von Figur und Persönlichkeit kann gezeigt werden. Dies ist wirkungsästhetisch im nichtprofessionellen Theater oft effektiver. Das Verschwinden der Nahtstelle ist an viele technische Erfahrungen und Übungen gebunden, über die Patienten zumindest am Beginn nicht verfügen. Allzu schnell spielten sie nur eigene Erfahrungen oder würden sich auf der Bühne in sich selbst verfangen. Banalster peinlicher Realismus wäre das Ergebnis, das den Patienten nicht schützt, sondern an sich selbst ausliefert.

Theater und Therapie sind Krisen. Theater erlebt und bewältigt sie im Als-ob-Modus. Therapie versucht, die Wirklichkeit eines krisengeschüttelten Individuums $\mathrm{zu}$ heilen und wieder lebbar und erlebbar zu gestalten. Ersteres agiert in der Fiktion. Zweiteres in der schnöden anstrengenden Wirklichkeit, die für den Patienten oft kaum zu ertragen ist.

In Lebenssituationen, die krisenhaft und niederschmetternd erfahren werden, mit neuen Krisen heilen zu wollen, scheint nicht nur paradox. Es ist es. Aber gerade die Paradoxien des Theaters als ein im Produzieren ständig mit sich selbst ringender Prozess können heilende Potenzen entfalten. Der Weg ist nicht die Entschärfung der Widersprüche, sondern ihr konsequentes Ausschreiten, im gewissen Sinne ihre Verschärfung. Nun muss aber genau diese Verschärfung im geschützten Raum geschehen. Einem geschützten Raum, der als solcher aber nicht von den Patienten erfahren wird. Für sie gelten vielmehr die Echtzeitbedingungen jedes normalen Theaters. Das heißt, sich mit sich selbst, vielen Verabredungen und Disziplinen in einem entschei- 
denden Moment der Öffentlichkeit preiszugeben. Dieser Moment fokussiert alle in den Probenprozessen gemachten Erfahrungen und die gesamte Persönlichkeit des Patienten in den Brennpunkt der Blicke der Zuschauenden. Der Patient durchschreitet starke Exzentrizität, um in der Mitte der Normalität anzukommen. Dies darf nicht zur Leugnung der eigentlichen Position verkommen. Die Lebenssituation des Patienten muss in der Perspektive der Inszenierung vorkommen, damit die Inszenierung an dieser Stelle ehrlich zu den eigenen Schauspielern und den Zuschauern sein kann.

Die Krise und der Skandal sind dem Theater eingeschrieben. Die Therapie agiert mit und in der Krise und muss den Skandal meiden. Der Therapeut will die Kontingenz des menschlichen Leids aus der Krise führen. Der Dramatiker will die Krise zeigen, um Imagination zu provozieren und Darüberhinausgehendes zu provozieren. Beide treffen sich in einem Begriff von Krise, der sich als Chance versteht. Dies ist die Basis für Verstehen im Ringen. Empathie setzt immer das Aufeinandertreffen voraus. Theater und Therapie haben darin ihre substanzielle Gemeinsamkeit. Der intensivste und damit entscheidende Moment dieser Krise ist die Begegnung mit dem Publikum. Jetzt wird öffentlich gemacht, was sich bislang nur den Blicken der Mitproduzierenden darbot. Bislang galt das alles nur als Spiel, war alles nur vorläufig. Nun fügt sich das immer nur Probierte zu seiner präsentierten Endgültigkeit. Die Zeit zieht sich auf einen explodierenden Augenblick zusammen. Jetzt wandelt sich das bisherige Als-ob für den Agierenden in eine dramatisch intensive Wirklichkeit. Jetzt ist der Moment gekommen, da die Stimme versagen, der Text vergessen, die verabredete Bewegung scheitern und/oder ein Schritt ins Leere gehen kann. Bis in die Leiblichkeit spürt jeder die Anspannung und die Verunsicherung.

Die Unsicherheit und das Risiko sind dem Theater durch seinen Live-Charakter eingeschrieben. Theater ist immer ein Ereignis, das den Zuschauer und den Spielenden braucht, um geschehen zu können.

Jede Vorstellung ringt um die Wiederholung des zuvor Erarbeiteten. So stemmt es sich der Flüchtigkeit entgegen. Theater versucht, den vergehenden Augenblick zu retten. Und weil es dies nicht kann, nicht einmal in der Lage ist, den Moment zu verlängern, ihn nicht in die Dauer dehnen kann, versucht es, ihn so genau wie möglich zu wiederholen. Daran scheitert jede Form von Theater, und dies ist die nichteinholbare Qualität und Faszination von theatralem Arbeiten in der Psychiatrie.

Theater akkumuliert in Wiederholungen den Probenprozess und wiederholt ihn in jeder weiteren Aufführung neu. Es versucht, die Flüchtigkeit des vergehenden Moments durch die Wiederholungen zu ignorieren. Es stemmt sich durch gemeinsam festgelegte Abläufe, durch ein technisches Gerüst, welches versucht, immer gleich zu bleiben, durch die fortlaufende Wiederholung der Verabredungen dagegen. Was einzig wirklich sich wiederholt, ist das Scheitern dieses Versuchs. In diesem Ringen transformiert sich der Live-Charakter in die Leibhaftigkeit der theatralen Momente.

Für die auf der Bühne Stehenden bergen diese Augenblicke existenzielle Risiken. Erfüllen sich ihre Ängste und bestätigen sich ihre Zweifel, wird das Darstellen auf der Bühne nicht nur Scheitern, sondern in peinliche Selbstenthüllung umschlagen. Sie geben sich dem Publikum anheim und sollten von dessen Reaktionen nicht fallengelassen, sondern wahrgenommen werden. Dies liegt allein im künstlerischen Gelingen. Dabei gilt nicht das reine Gefallen oder Missfallen in der Gunst des Publikums, sondern erst einmal nur die intensive theatralische Auseinandersetzung und ihre Präsentation. Und deswegen gilt unterhalb des Einspruchsrechtes des Therapeutischen die Autonomie des Ästhetischen. In ihr müssen die theatralische Konstruktion und die Spielführung des Regisseurs so stark sein, dass sie dem Spielenden nicht nur Schutz gewähren, sondern ihn erheben und auf ein Podest stellen. Der Spielende selbst schafft jetzt den glückhaften, berauschenden Moment des öffentlichen Gelingens. Durch ihn werden die Binnenperspektiven aufgebrochen und in der Präsentation nach außen gewendet. Die Therapie verwandelt sich jetzt in Theater, die Patienten in Darsteller und der Therapeut in einen Regisseur.

Die Proben ragen in das Regelgefüge der forensischen Klinik hinein, verändern vorübergehend die üblichen Normen. Zumindest unterbrechen sie es. Das ist Bedingung für das Gelingen der Inszenierung, da diese immer ,anders“ sein muss als das „Normale“. Abweichung von der üblichen Normalität kann auch Teil des therapeutischen Gelingens sein und führt oft zu Konflikten. Probenarbeit will und soll ein geschützter Raum des Andersseins bleiben können. Der Raum, in welchem die Probenarbeit sich entwickelt, muss seine Normalität und normierende Kraft behaupten können. Die Proben sind ein Möglichkeitsraum. Die Wirklichkeit davor und danach ist streng normiert. Fiktion steht gegen Klassifikation. Das führt zu organisatorischen, zeitlichen bis hin zu persönlichen Konflikten, die jederzeit gemanagt werden.

Die Vermittlungsarbeiten dürfen jedoch nicht mitteln. Sie bleiben geprägt und geleitet von einer künstlerischen Idee, von dem Spiel, was auf den Proben entsteht. Diese Idee bestimmt, in welchen Inszenierungszusammenhängen der Patient, der jetzt ein Schauspieler ist, später in aller Öffentlichkeit agiert. Jene Idee zwingt den Spieler in die öffentlichen Blicke vieler schauenden Menschen. Trotzdem ist sie ein schützender Kokon, der sich um die Seele des Spielenden legt, während sie ihn gleichzeitig antreibt, sich fremden Blicken zu präsentieren. Sie verlangt von dem Spielenden, Scham zu überwinden, um so öffentlich zu sein wie noch nie zuvor im Leben. Die künstlerische Idee des Regisseurs muss dominieren und sich im selben Moment als Diener des 
Schauspielers verstehen. Sie muss im Schauspieler selbst die Möglichkeiten ihrer Realisierung entdecken und mit diesen in ihm selbst liegenden Potenzialen seine bisherigen Erfahrungen überschreiten, um etwas zu schaffen, das zuvor nicht in ihm lag.

Im Bangen vor der Premiere, in der letztlich existenziellen Leidenschaft, sich vor Publikum im Dienste einer Geschichte selbst zu zeigen, verwandelt der Darsteller die Therapie in den einzigartigen theatralen Moment, den er den Zuschauern schenkt und wofür sie sich bei ihm mit Applaus bedanken. Mehr intensives Theater ist in dieser Situation kaum denkbar. Und in einer gewissen Weise kommt Theater hier bei sich selber an. Auf intensive Weise wird es Therapie.

Funding Open Access funding provided by Projekt DEAL.

Interessenkonflikt F. Triebenecker und S. Orlob geben an, dass kein Interessenkonflikt besteht.

Open Access Dieser Artikel wird unter der Creative Commons Namensnennung 4.0 International Lizenz veröffentlicht, welche die Nutzung, Vervielfältigung, Bearbeitung, Verbreitung und Wiedergabe in jeglichem Medium und Format erlaubt, sofern Sie den/die ursprünglichen Autor(en) und die Quelle ordnungsgemäß nennen, einen Link zur Creative Commons Lizenz beifügen und angeben, ob Änderungen vorgenommen wurden.

Die in diesem Artikel enthaltenen Bilder und sonstiges Drittmaterial unterliegen ebenfalls der genannten Creative Commons Lizenz, sofern sich aus der Abbildungslegende nichts anderes ergibt. Sofern das betreffende Material nicht unter der genannten Creative Commons Lizenz steht und die betreffende Handlung nicht nach gesetzlichen Vorschriften erlaubt ist, ist für die oben aufgeführten Weiterverwendungen des Materials die Einwilligung des jeweiligen Rechteinhabers einzuholen.

Weitere Details zur Lizenz entnehmen Sie bitte der Lizenzinformation auf http://creativecommons.org/licenses/by/4.0/deed.de.

\section{Literatur}

Orlob S, Gillner M, Riedel S, Lübcke-Westermann D (1998) Kunsttherapie in der Forensischen Psychiatrie. Z Strafvollzug Straffälligenh 47:163-166

Triebenecker F (2018) Theater spielen heilt. Klett-Cotta, Stuttgart

Wißner A (2018) Gefängnistheater. Monatsschr Kriminol Strafrechtsreform 101:484-496 